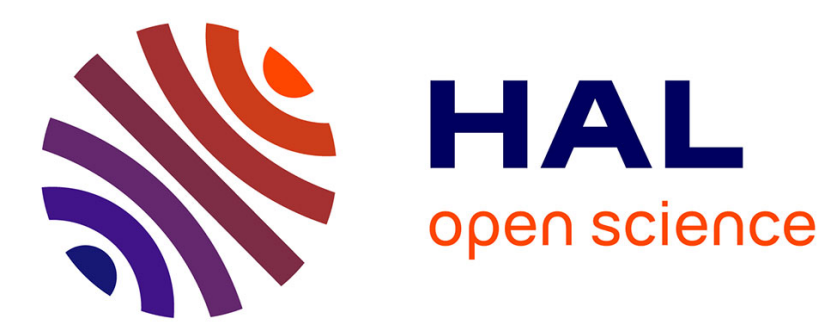

\title{
ETUDES EN SOLUTION DE MACROMOLECULES BIOLOGIQUES A LURE
}

\author{
P. Vachette, A. Tardieu, F. Seydoux, C. Pontillon, M. Lemaire, B. Krop, B. \\ Juillemier, A. Gulik, F. Rousseaux, M. Lemonnier
}

\section{- To cite this version:}

P. Vachette, A. Tardieu, F. Seydoux, C. Pontillon, M. Lemaire, et al.. ETUDES EN SOLUTION DE MACROMOLECULES BIOLOGIQUES A LURE. Journal de Physique Colloques, 1980, 41 (C3), pp.C3-281-C3-282. 10.1051/jphyscol:1980345 . jpa-00219864

\section{HAL Id: jpa-00219864 https://hal.science/jpa-00219864}

Submitted on 1 Jan 1980

HAL is a multi-disciplinary open access archive for the deposit and dissemination of scientific research documents, whether they are published or not. The documents may come from teaching and research institutions in France or abroad, or from public or private research centers.
L'archive ouverte pluridisciplinaire HAL, est destinée au dépôt et à la diffusion de documents scientifiques de niveau recherche, publiés ou non, émanant des établissements d'enseignement et de recherche français ou étrangers, des laboratoires publics ou privés. 
ETUDE DE LA DISPERSION ANOMALE DES RAYONS $X$ PAR LA DIFFUSION CENTRALE

Une solution de ferritine a été choisie pour étudier l'effet de la dispersion anomale en utilisant les rayons $X$ émis par $l$ 'anneau de stockage DORIS du Deutsches Elektronen-synchrotron (DESY). Les mesures ont été faites avec un instrument (X15) pour la diffusion aux petits angles dans le laboratoire de 1 'antenne de LEBM à Hambourg.

Le faisceau monochromatique produit par deux monocristaux de germanium en rêflexion 111 était diffusé par une solution de $10 \%$ de ferritine. Un détecteur avec une résolution spatiale en deux dimensions, qui a été construit par A.Gabriel, permettait la collection de tous les photons diffusés.

La demi-hauteur de la diffusion centrale a une allure régulière sauf près du bord de l'absorption de fer. La dépression de la demi-hauteur est environ $5 \%$ près de longueur d'onde de $1.73 \AA$. Ce résultat est basé sur 80 expériences avec des longueurs d'ondes différentes entre 1.5 et $2.0 \AA$. Les expériences ont encore un caractère préliminaire et seront répétés pour améliorer la précision statistique.

\section{H.B. ST UHRMANN}

Laboratoire Européen de la Biologie Moléculaire, Hambourg

CHANGEMENTS STRUCTURAUX LORS DE LA CONTRACTION DU MUSCLE RETRACTEUR DE MYTILUS VUS PAR MESURES DE DIFFRACTION RAPIDES

Les variations d'intensité de la réflexion équatoriale à $120 \AA$ et de la réflexion méridionale à $145 \AA$ dans le diagramme de diffraction du muscle rétracteur antérieur de Mytilus ont été étudiées au cours de la contraction isométrique et au cours de la "catch contraction". Une caméra à optique focalisante (miroirs et monochromateur) a été utilisêe à 1 'anneau de stockage DORIS. Cette caméra (Hendrix, Koch, Bordas, J.App1.Cryst. sous presse) permet d'obtenir de $1^{\prime}$ ordre de $10^{11}$ photons/sec dans un foyer de $1 \mathrm{~mm}^{2}$. Elle est équipée d'un détecteur à ligne à retard construit par A.Gabriel.
Les résultats obtenus avec une résolution en temps de 1 'ordre de $100 \mathrm{msec}$ démontrent pour la première fois la relation directe entre le nombre de têtes de myosine attachées aux filaments secondaires et la tension dans le muscle.

M.H.J.KOCH et J.BORDAS

EMBL c/O DESY, Notkestrasse 85, D-2000 Hambourg 52, RFA

\section{J.LOWY et F.R.POULSEN}

The Open University, Oxford Research Unit, Foxcombe Hall, Berkeley Road, Boars Hill, Oxford, GB

ETUDES EN SOLUTION DE MACROMOLECULES BIOLOGIQUES A LURE

Le montage de diffusion centrale de LURE est maintenant opérationnel pour l'étude de macromolécules biologiques en solution. Comme ces préparations ont un pouvoir diffusant très faible et ne sont souvent disponibles qu'en petites quantités, la géométrie du montage a été choisie pour permettre de travailler avec des échantillons de faibles dimensions et pour optimiser le rapport signal sur bruit. Les performances de l'installation sont équivalentes à celles des meilleurs montages de laboratoire, avec un gain en intensitê de l'ordre de 100 à 1000 .

Au cours de l'année écoulée, plusieurs séries d'expériences ont porté sur l'étude par la méthode de variation de contraste de la structure de systèmes macromoléculaires complexes, comprenant divers composants -protēines, acides nucléiques, lipides-. L'analyse de courbes de diffusion -rayons $\mathrm{X}$ ou neutrons- enregistrées en fonction de la densité du solvant constitue l'une des méthodes les plus puissantes pour étudier en solution la structure de ces systèmes. Nous avons entrepris un tel type d'étude sur les sous-unités du ribosome d'E. Coli, ainsi que sur les "core particles" de chromatine de foie de rat, en utilisant le saccharose pour faire varier la densité êlectronique du so1vant. L'intensité du faisceau fourni par DCI permet d'obtenir une courbe d'intensité en $15 \mathrm{mn}$ environ, ce qui permet d'enregistrer le même jour 10 à 20 courbes à partir de la même préparation. L'interprétation des données recueillies est en cours.

Par ailleurs, diverses études structurales 
de protéines ont été poursuivies :

- L'étude morphologique de l'ATPase Ca dépendante du reticulum sarcoplasmique solubilisée en déoxycholate fait apparaître la protéine comme une particule allongée de diamètre maximal $115 \pm 5 \AA$.

- La morphologie des protêines ribosomales est une question qui a donné lieu à de nombreuses controverses. Nous avons amorcé des collaborations (A.M. Freund, I.B.M.C. Strasbourg, J.Dijk et J.Littlechild, M.P.I. Für Molekülare Genetik, Berlin) pour étudier ou réétudier quelques unes de ces protéines, préparées selon deux méthodes différentes, et dans diverses conditions de concentration et de force ionique. Les expériences ne sont pas encore entièrement analysées, mais les premiers dépouillements montrent qu'il est possible de mesurer le rayon de giration de protéines de masse moléculaire 10000 à des concentrations de 2 à $4 \mathrm{mg} / \mathrm{ml}$ en une heure d'exposition environ.

- La détermination des bases structurales des mécanismes de contrôle allostérique est un problème que $I$ 'on peut aborder par la diffusion des rayons- $X$ en solution. En effet, parmi les nombreuses techniques, notamment spectroscopiques, qui ont été utilisées, pratiquement aucune $n$ 'est spécifiquement sensible aux changements de structure quaternaire. En revanche les spectres de diffusion des rayons- $X$ en solution peuvent fournir plusieurs paramètres -par exemple le rayon de giration- qui dépendent essentiellement de la structure quaternaire. L'étude des changements du spectre de diffusion d'une enzyme après saturation par le substrat peut finalement permettre de détecter l'existence d'états quatermaires transizoires autres que les formes extrêmes $T$ et $R$. Enfin il est possible, grâce à l'intensité du rayonnement synchrotron, d'envisager l'étude de za cinétique de transconformation. Nous avons entrepris une étude de la transition allostérique de la phosphofructokinase de levure (PFK). Les premiers résu1tats montrent que la courbe entière, qui présente plusieurs accidents caractéristiques, est nettement modifiée après fixation du substrat. L'étude des transitions allostériques, sur la PFK ou sur d'autres enzymes, devrait être activement poursuivie au cours de l'année prochaine.

P.VACHETTE, A.TARDIEU, F.SEYDOUX ${ }^{+}$, C.PONTILLON, M.LEMAIRE, B.KROP, B.JULLEMIER, A.GULIK et F. ROUSSEAUX, M. LEMONNIER* Centre de Génétique Moléculaire, CNRS, 91190 Gif-
sur-Yvette

* LURE, 91405 Orsay

+Laboratoire d'Enzymologie Physico-Chimique et Moléculaire, 91405 Orsay (Décédé le 25 avril 1979)

STRUCTURE TRIDIMENSIONNELLE DES PROTEINES ET RAYONNEMENT SYNCHROTRON : ACQUIS ET PERSPECTIVES

\section{A MOYEN TERME}

Le rayonnement $X$ êmis par l'anneau de collisions DCI a été utilisé depuis 1976 à LURE par diverses équipes françaises et étrangères pour enregistrer les spectres de diffraction de protéines cristallisées et, dans certains cas, de dérivés isomorphes. Ces données sont - ou seront - utilisées soit pour déterminer la structure tertiaire de ces molécules (ex : arginine kinase, pepsine, facteur de croissance du nerf) soit pour en reprendre l'étude sous résolution plus êlevée (ex : tyrosyl t-RNA synthêtase, phosphorylase b). Le montage expérimental délivre un rayonnement $x$ monochromatique focalisé linéairement, de longueur $d$ 'onde ajustable entre $\sim 1-2,6 \AA$; les enregistrements sont effectués par la méthode d'oscillation à 1 'aide de cassettes photographiques planes, en " $V$ " ou semi-cylindriques. La source synchrotron offre ici des caractéristiques quasi-idéales : brillance élevée, faible divergence du faisceau, continuité spectrale, stabilité. En prenant pour référence un tube à anticathode tournante, les temps d'exposition sont réduits d'un facteur 50-100, le rapport signal/bruit est accru et la résolution spatiale est comparable à celle que permet d'obtenir un montage usuel à double monochromateur; le débit de dose plus élevé permet, en règle générale, de collecter plus d'information avant dégradation sous irradiation pour un cristal donné. Le gain global est particulièrement remarquable pour les cristaux à grande maille cristalline étudiés sous haute résolution et pour les cristaux de faibles dimensions et/ou de qualité cristalline médiocre.

Dans une seconde étape, 1'équipement actuel sera doublé par un système d'acquisition avec détecteur électronique localisant les photons $x$, actuellement en essais. Ce diffractomètre multirêflexion permettra la collection rapide de données de diffraction (des mesures à basses températures de structures dans des états transitoires peuvent être envisagées) ; il facilitera aussi l'applica- 